\title{
Multi-epoch VLBA observations of radio galaxy 0932+075: is this a compact symmetric object?
}

\author{
Andrzej Marecki ${ }^{1}$ and Aleksandra Sokołowska ${ }^{1,2}$ \\ 1 Toruń Centre for Astronomy, Faculty of Physics, Astronomy and Informatics, Nicolaus Copernicus University, Toruń, Poland \\ e-mail: amr@astro.uni.torun.pl \\ ${ }^{2}$ Institute for Computational Science, University of Zurich, Winterthurerstrasse 190, 8057 Zurich, Switzerland \\ e-mail: alexs@physik.uzh.ch
}

Received 15 July 2013 / Accepted 4 July 2014

\begin{abstract}
A part of the radio structure of the galaxy $0932+075$ emerged as a possible compact symmetric object (CSO) after the observation with the Very Long Baseline Array (VLBA) at $5 \mathrm{GHz}$ in 1997. More than a decade later, we carried out observations at 5, 15.4, and $22.2 \mathrm{GHz}$ using the VLBA to test this possibility. We report here that we have found a component whose spectrum is inverted in the whole range from $5 \mathrm{GHz}$ to $22 \mathrm{GHz}$ and we label it a high-frequency peaker (HFP). Using a set of $5 \mathrm{GHz}$ images from two epochs separated by 11.8 years and a set of $15.4 \mathrm{GHz}$ images separated by 8.2 years, we were able to examine the proper motions of the three components of the CSO candidate with respect to the HFP. We found that their displacements cannot be reconciled with the CSO paradigm. This has led to the rejection of the hypothesis that the western part of the arcsecond-scale radio structure of 0932+075 is a CSO anchored at the HFP. Consequently, the HFP cannot be labelled a core and its role in this system is unclear.
\end{abstract}

Key words. radio continuum: galaxies - galaxies: active - galaxies: individual: 0932+075

\section{Introduction}

The term compact symmetric object (CSO) was introduced by Readhead et al. (1994) and Wilkinson et al. (1994) to label three objects from the survey of 65 very compact radio sources observed using Very Long Baseline Interferometry (VLBI) at $5 \mathrm{GHz}$ (Pearson \& Readhead 1988). The authors pointed out that despite their small sizes $(\lesssim 1 \mathrm{kpc})$ CSOs were symmetric and in this regard they resembled much larger radio sources. The nature of CSOs could be explained in two ways that were immediately taken into consideration by Readhead et al. (1994): CSOs are either precursors of standard large-scale doubles or they constitute a class of short-lived objects that decay too quickly to make it possible for them to evolve from subkiloparsec-sized structures to more extended forms. Both options belong to the so-called youth scenario of compact sources. It appears that since 1994, when the CSO class was recognised, the youth scenario has been valid (see e.g. Fanti 2009, for a review).

The most straightforward proof that CSOs are young is based on the measurements of kinematic ages calculated from their lobe expansion velocities extracted from multi-epoch VLBI observations. These measurements were carried out by Owsianik \& Conway (1998), Owsianik et al. (1998), Taylor et al. (2000), Marecki et al. (2003), Polatidis \& Conway (2003), Gugliucci et al. (2005), Nagai et al. (2006), Polatidis (2009), and An et al. (2012). To date, the lobe expansion velocities, or at least their lower limits, have been found for 37 CSOs and so their kinematic ages, or at least their upper limits, could have been estimated. Given that for 27 of them the redshifts are known, both lobe expansion velocities and the kinematic ages of the sources can be calculated properly, i.e. with the time-dilation factor taken into account. They happen to be less than 3000 years old, but a considerable fraction - eleven CSOs out of the twenty-seven are younger than 500 years, which is a meaningful overabundance pointed out by Gugliucci et al. (2005). This circumstance supports the conjecture that the evolution of the classic double radio sources often comes to a premature end at the CSO stage as suggested by Readhead et al. (1994).

Since CSOs are an astrophysically important albeit not very numerous class of objects, the original idea of the investigation presented here was to test whether $0932+075$ (JVAS J0935+0719), a radio source identified with a galaxy at $z=0.29$ that has not yet been recognised as a CSO in the literature, could in fact be another CSO. The scientific rationale of such an endeavour was that $0932+075$ was mapped with the VLA at $8.4 \mathrm{GHz}$ in the course of the Jodrell Bank-VLA Astrometric Survey (JVAS; Browne et al. 1998) and then included in JVAS although with a caveat that it was not a pointlike source but an asymmetric double whose components are separated by $\sim 0$. 4 . It was therefore selected as a gravitational lens (GL) candidate and followed up with MERLIN at $5 \mathrm{GHz}$ in the course of the Cosmic Lens All-Sky Survey (CLASS; Myers et al. 2003). These observations confirmed that $0932+075$ was a 0.38 -wide, i.e. $1.6 \mathrm{kpc}$-wide ${ }^{1}$ double. As such, it was still a GL candidate and was re-observed at $5 \mathrm{GHz}$ with the VLBA on 02 August 1997 (epoch 1997.6). Based on the results of that observation, the possibility of the presence of a GL system appeared unlikely because the surface brightness values of the two major components were highly unequal: their ratio was 22.6:1 (Browne et al. 2003). However, a new circumstance emerged at this point and subsequently became the cornerstone of the

1 For redshift $z=0.29$ and the standard cosmological parameters $\left(H_{0}=71 \mathrm{~km} \mathrm{~s}^{-1} \mathrm{Mpc}^{-1}, \Omega_{\mathrm{m}}=0.27, \Omega_{\Lambda}=0.73\right)$, the angular distances pertinent to $0932+075$ should be multiplied by a factor of $4.317 \mathrm{pc} / \mathrm{mas}$ to be converted to projected linear distances. 

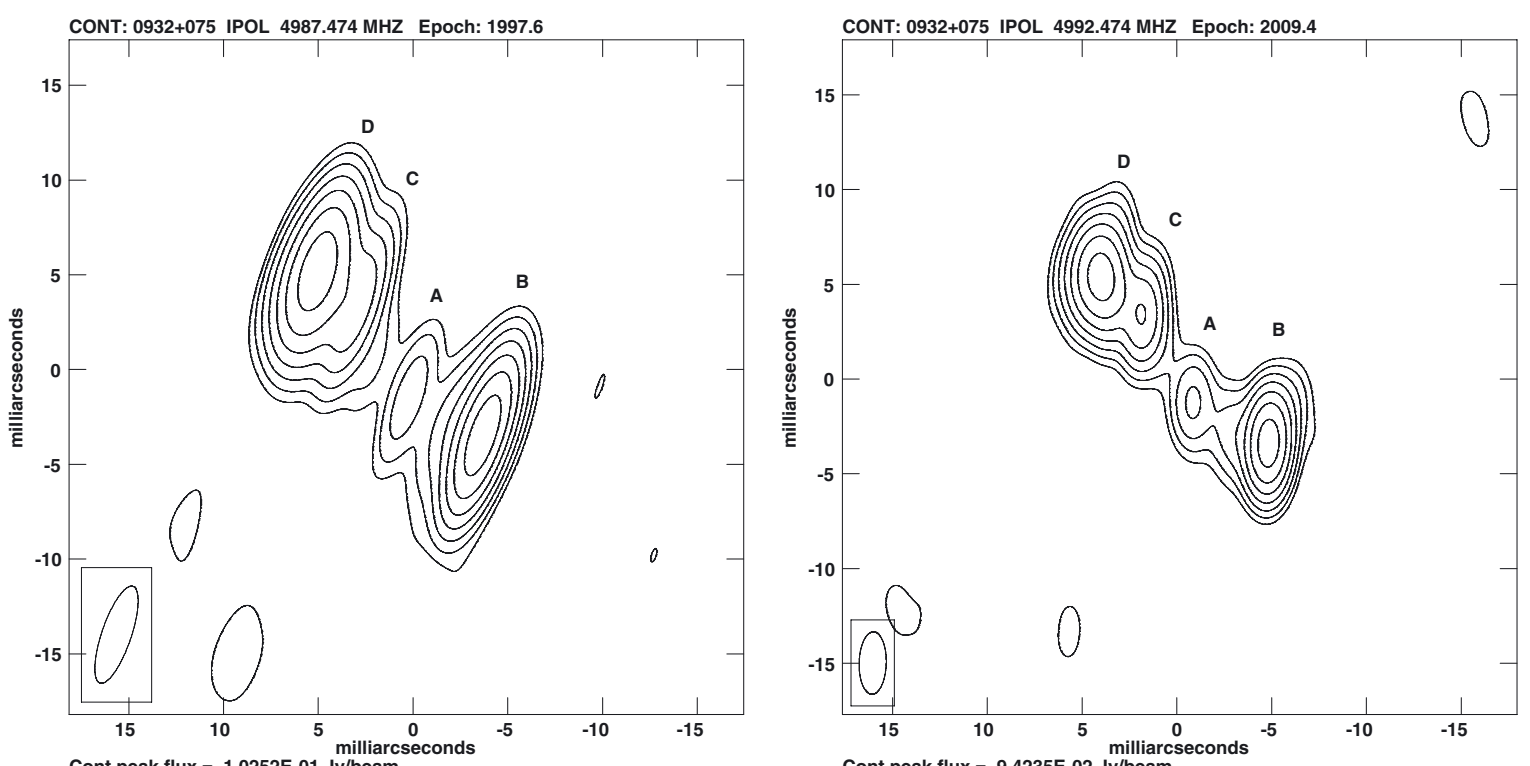

Cont peak flux $=1.0252 E-01$ Jy/beam
Levs $=1.000 E-03 *(1,2,4,8,16,32,64,128)$
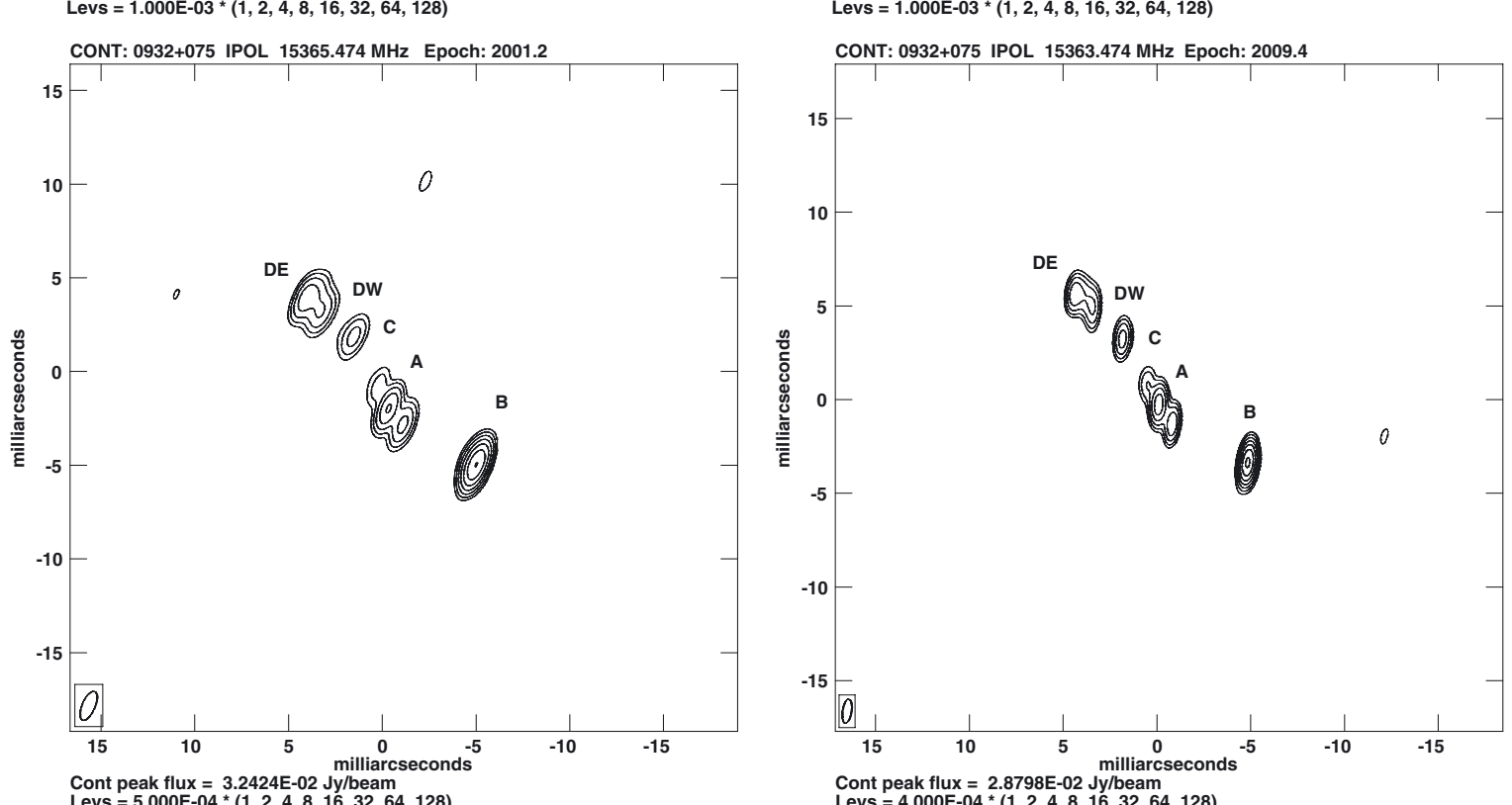

Fig. 1. Western part of $0932+075$ as seen with the VLBA. In all panels, the contours are increased by a factor of 2. Upper left: image resulting from the $5 \mathrm{GHz}$ observation carried out on 02 August 1997 and reported in Browne et al. (2003). The first contour level corresponds to $1 \mathrm{mJy} / \mathrm{beam}$. The beam size is $5.4 \times 1.6$ mas at the position angle of $-19^{\circ}$. Upper right: image resulting from the $5 \mathrm{GHz}$ observation that we carried out on 17 May 2009. The first contour level corresponds to $1 \mathrm{mJy} / \mathrm{beam}$. The beam size is $3.3 \times 1.4$ mas at the position angle of $-1.6^{\circ}$. Lower left: image resulting from the observation carried out at $15.4 \mathrm{GHz}$ on 22 March 2001. The first contour level corresponds to $0.4 \mathrm{mJy} / \mathrm{beam}$. The beam size is $1.7 \times 0.7$ mas at the position angle $-23^{\circ}$. Lower right: image resulting from the $15.4 \mathrm{GHz}$ observation that we carried out on $17 \mathrm{May} 2009$. The first contour level corresponds to $0.4 \mathrm{mJy} / \mathrm{beam}$. The beam size is $1.3 \times 0.5 \mathrm{mas}$ at the position angle of $-8.5^{\circ}$.

present work. Owing to the resolution attainable with the VLBA, the detailed structure of $0932+075$ was revealed (see Fig. 8 in Browne et al. 2003). In particular, the western feature shown in that image, i.e. the component that is $\sim 23$ times stronger than its eastern companion, turned out to be an almost symmetric compact double. Given its overall span $(\sim 50 \mathrm{pc})$, it was justified to tentatively label the western part of $0932+075$ a CSO. Interestingly, the position angle of the potential $\mathrm{CSO}\left(45^{\circ}\right)$ is not the same as that of the line connecting the two arcsecond-scale components $\left(60^{\circ}\right)$.

The raw observational data acquired by Browne et al. (2003) belongs to the public domain. We took this opportunity and carried out a standard reduction procedure of that data in AIPS independently. The structure of the CSO-like part that we have obtained is shown in the upper-left panel of Fig. 1. In that image, three conspicuous components: A, B, and D and at least one more, $\mathrm{C}$, are visible, although $\mathrm{C}$ is blended with its stronger neighbouring component $\mathrm{D}$. The interpretation of the three wellresolved components is not obvious, however. While the dominant features at the source's extremities are likely to be the lobes, the nature of the third one between them is unclear; it could be the core, but this is only a hint that cannot be regarded as a piece of evidence in favour of such a conjecture. Nevertheless, since the suggestion that the structure shown in the upper-left panel of Fig. 1 could be a CSO seemed reasonable, we launched an observing programme to either prove it or reject it. 
A. Marecki and A. Sokołowska: Is radio galaxy $0932+075$ a compact symmetric object?

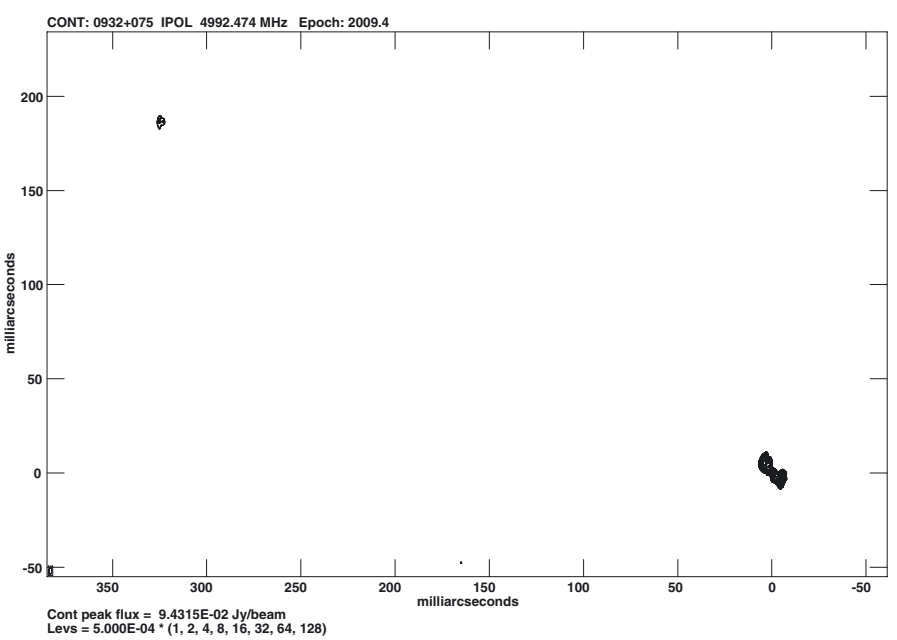

Fig. 2. Complete image of $0932+075$ at $5 \mathrm{GHz}$ resulting from the observation we carried out on 17 May 2009. The first contour level corresponds to $0.5 \mathrm{mJy} / \mathrm{beam}$. Contours are increased by a factor of 2 .

\section{New high-resolution observations}

We carried out the second-epoch observations of $0932+075$ with the VLBA at two frequencies, $5 \mathrm{GHz}$ and $15.4 \mathrm{GHz}$, on 17 May 2009 (epoch 2009.4), i.e. 11 years and 9.5 months after the first epoch. Sixty-four-MHz bandwidth was sampled at both frequencies (LHC polarisation). The first frequency was chosen for the sake of compatibility with the data used by Browne et al. (2003) so that possible proper motions of the components could be found, whereas the observation at the second was requested to estimate spectral indices aiming at identification of the putative core. The source $0932+075$ was observed for $80 \mathrm{~min}$ at each frequency. No phase referencing was applied ${ }^{2}$ as the source was strong enough to obtain the fringe residual delays and rates using its own visibilities. Standard continuum processing was applied. The resulting images are presented in Figs. 1 and 2. The $5 \mathrm{GHz}$ image in Fig. 2 is a complete view of the source. It encompasses both its parts: the weak and diffuse eastern component and the dominant western one. Figure 2 is an equivalent of Fig. 8 in Browne et al. (2003); it shows exactly the same part of the sky at the same frequency, but in an 11.8-year later epoch. The western part of 0932+075 alone, as seen in epoch 2009.4, is shown in the upper-right $(5 \mathrm{GHz})$ and lower-right $(15.4 \mathrm{GHz})$ panels of Fig. 1.

In the epoch $2009.45 \mathrm{GHz}$ image, the western part of $0932+075$ consists of four well-resolved components. The outer two are dominating and quite symmetric - their flux densities amount to 100 and $132 \mathrm{mJy}$ (see Table 1) - hence the CSO nature of the western part of $0932+075$ seems to be plausible at first sight. The interpretation of the inner two components in that image is not straightforward though: either one of them is the core or neither is. The $5 \mathrm{GHz}$ and $15.4 \mathrm{GHz}$ flux densities of C in epoch 2009.4 are $44 \mathrm{mJy}$ and $5.4 \mathrm{mJy}$, respectively (see Table 1), which means that $\mathrm{C}$ can be excluded as a possible core due to its very steep spectrum. Next, we investigate whether the core is associated with the component A. However, while unresolved at $5 \mathrm{GHz}$, this component reveals a triple structure at $15.4 \mathrm{GHz}$, so at that stage the location of the core remains an open matter.

Figure 3 presents a magnified view of the A-complex extracted from our $15.4 \mathrm{GHz}$ image in Fig. 1 (lower-right panel). We overlaid the $5 \mathrm{GHz}$ and $15.4 \mathrm{GHz}$ images of the epoch 2009.4

\footnotetext{
2 Browne et al. did not use phase referencing either.
}

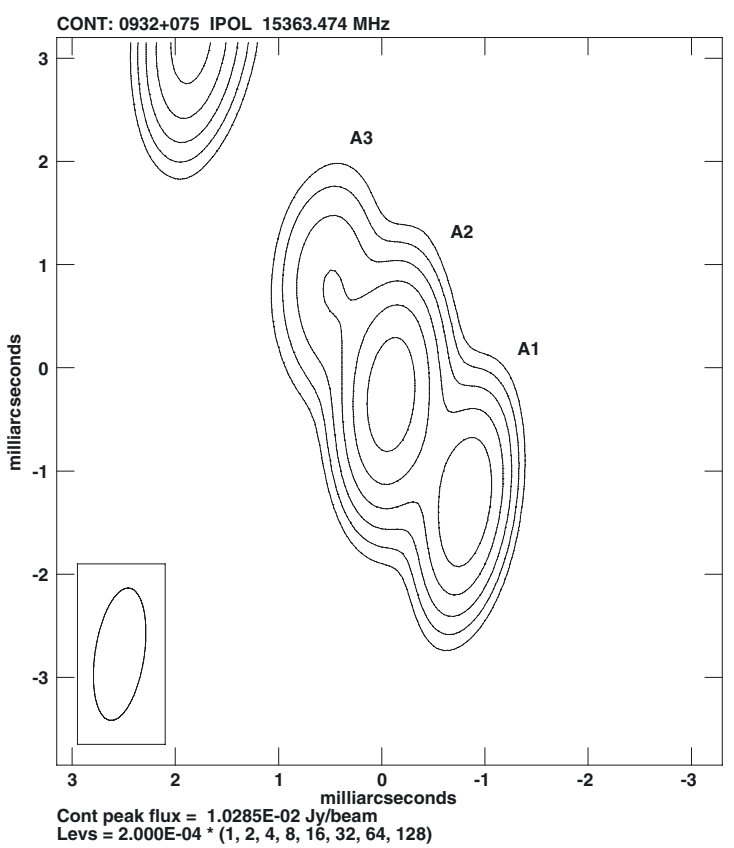

Fig. 3. Enlarged cutout from the $15.4 \mathrm{GHz}$ image shown in Fig. 1 (lower-right panel) centred on the A-complex.

Table 1. Flux densities of the components of the western part of 0932+075 [mJy].

\begin{tabular}{lrrrrr}
\hline \hline Epoch & 1997.6 & 2009.4 & 2001.2 & 2009.4 & 2011.0 \\
\hline Component & 5.0 & 5.0 & 15.4 & 15.4 & 22.2 \\
\hline A & 7.1 & 11 & - & - & - \\
A1 & - & - & 6.8 & 6.6 & 8.3 \\
A2 & - & - & 9.3 & 12 & 31 \\
A3 & - & - & 4.0 & 3.7 & $-a$ \\
B & 108 & 100 & 36 & 32 & 28 \\
C & 37 & 44 & 3.9 & 5.4 & 5.2 \\
D & 141 & 132 & - & - & - \\
DW & - & - & $-b$ & 8.2 & 6.4 \\
DE & - & - & 11 & 8.4 & 5.1 \\
\hline
\end{tabular}

Notes. ${ }^{(a)}$ Flux density of the component A3 could not be measured reliably because of blending with neighbouring component A2. ${ }^{(b)}$ Flux density of the component DW could not be measured reliably because of blending with neighbouring component DE.

to identify the respective components using $\mathrm{B}$ as a reference point because of its compactness. As a result, the positions of $\mathrm{C}$ at both frequencies matched well, the position of $\mathrm{D}$ at $5 \mathrm{GHz}$ was halfway between DE and DW at $15.4 \mathrm{GHz}$, whereas the component $\mathrm{A}$ at $5 \mathrm{GHz}$ matched neither $\mathrm{A} 2$ nor $\mathrm{A} 3$, but its position agreed with that of $\mathrm{A} 1$ at $15.4 \mathrm{GHz}$.

To confirm this finding quantitatively, we fitted Gaussian profiles to $\mathrm{A}$ and $\mathrm{B}$ at $5 \mathrm{GHz}$ as well as to A1, A2, A3, and $\mathrm{B}$ at $15.4 \mathrm{GHz}$ using AIPS task JMFIT. We found that the separations $\mathrm{B}-\mathrm{A}$ at $5 \mathrm{GHz}$ and $\mathrm{B}-\mathrm{A} 1$ at $15.4 \mathrm{GHz}$ were the same (4.5 mas) while the separations $\mathrm{B}-\mathrm{A} 2$ and $\mathrm{B}-\mathrm{A} 3$ were 5.7 mas and 6.6 mas, respectively. We assume that the uncertainties of these figures amount to $20 \%$ of the beam size ${ }^{3}$. Clearly then,

3 This is much more than the formal position errors given by JMFIT; JMFIT, however, does not take into account either the density of the $u-v$ plane coverage, or the errors of the individual visibilities. The actual errors are very difficult to estimate hence our very conservative assumption of their magnitude. 


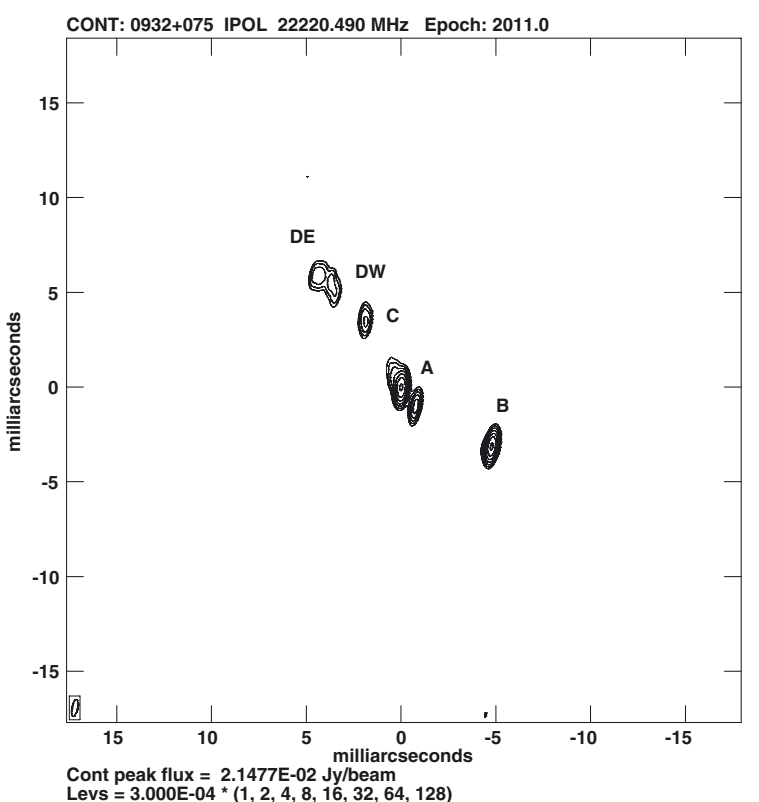

Fig. 4. Image resulting from the observation carried out at $22.2 \mathrm{GHz}$ on 15 December 2010. The first contour level corresponds to $0.3 \mathrm{mJy} / \mathrm{beam}$. The beam size is $0.9 \times 0.3$ mas at the position angle of $-11^{\circ}$.

component A seen at $5 \mathrm{GHz}$ is not a counterpart of either A2 or A3 seen at $15.4 \mathrm{GHz}$. This immediately raises the question whether any counterpart of A2 is present in the $5 \mathrm{GHz}$ map. It appears that there is no trace of such a counterpart in the $5 \mathrm{GHz}$ image, although in principle it could exist because, given the separation of 1.2 mas between $\mathrm{A} 2$ and $\mathrm{A} 1$ as measured at $15.4 \mathrm{GHz}$, the resolution attained at $5 \mathrm{GHz}$ is sufficient to resolve these two components at least partly. It follows that the flux density of A2 is too low at $5 \mathrm{GHz}$ to make it visible hence its spectrum between $5 \mathrm{GHz}$ and $15.4 \mathrm{GHz}$ is surely inverted. It could thus be expected that it might also be inverted towards higher frequencies.

To test this conjecture, we observed the western part of $0932+075$ with the VLBA at $22.2 \mathrm{GHz}$. The observation was carried out on 15 December 2010 (epoch 2011.0) covering a $128 \mathrm{MHz}$ bandwidth (LHC polarisation). The target was observed for $220 \mathrm{~min}$, again without phase referencing as the source was strong enough to obtain the fringe residual delays and rates using its own visibilities. Standard continuum processing was applied. The resulting image is shown in Fig. 4.

Finally, we searched the NRAO archive to find whether there existed any VLBA observations of $0932+075$ other than those by Browne et al. (2003) and ours. We found that $0932+075$ had been observed at $15.4 \mathrm{GHz}$ on 22 March 2001 (epoch 2001.2), but the outcome of that observation was never published (see Acknowledgements for details). The total on-source time was $\sim 70$ min for $0932+075$ at $32 \mathrm{MHz}$ bandwidth with LHC polarisation only. We processed the public-domain raw data acquired in the course of that observation in a standard way. The resulting image is shown in Fig. 1 (lower-left panel).

We measured the flux densities of the components of $0932+075$ in all five maps shown in Figs. 1 and 4 using AIPS task JMFIT. We assumed 5\% uncertainties of these measurements. Table 1 comprises the results along with the respective epochs to clarify which measurements are quasi-simultaneous and which are not. This is essential when trying to estimate the spectral indices because of the possible variability of the source's components which, if occurring, would complicate the interpretation of the results. However, regardless of the magnitude

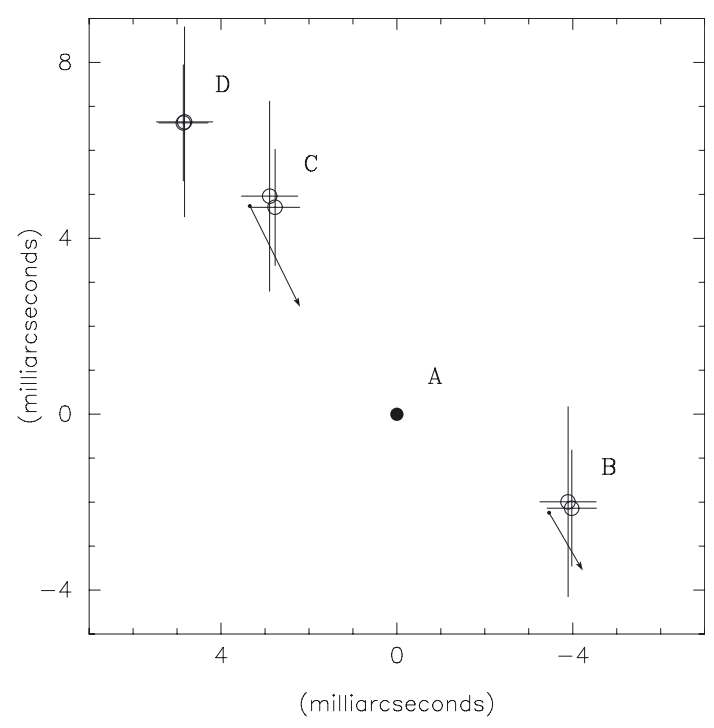

Fig. 5. Schematic diagram showing the positions of the four components seen in the $5 \mathrm{GHz}$ images shown in Fig. 1. Open circles represent the positions of the centroids of $\mathrm{B}, \mathrm{C}$, and $\mathrm{D}$ with respect to $\mathrm{A}$ in the epochs 1997.6 and 2009.4. Error bars correspond to $20 \%$ of the beam sizes. The arrows indicate the direction of the components' displacements. The length of each arrow is proportional to the magnitude of the respective displacement.

of variability of A2 between the epochs 2009.4 and 2011.0, it clearly has an inverted spectrum between $15.4 \mathrm{GHz}$ and $22 \mathrm{GHz}$ hence it most likely is the core. Combined with the unmeasurable $5 \mathrm{GHz}$ flux of A2, it appears that the flux density of A2 rises sharply in the whole range from $5 \mathrm{GHz}$ up to $22 \mathrm{GHz}$. Owing to this type of spectrum, A2 could be classified as a high-frequency peaker (HFP; see the definition of that class in Dallacasa et al. 2000).

\section{Measurements of proper motions}

After we found the most likely candidate for the core, we attempted a crucial test of the CSO hypothesis: if the western part of 0932+075 was a CSO then like in most CSOs (see An et al. 2012, and references therein) we would expect expansion of the mini-lobes B and D. By the same token, the component $\mathrm{C}$ would be expected to travel towards the north-east, i.e. in a way analogous to D since both are on the same side of the putative core. We measured the changes of the angular distances of B, C, and $\mathrm{D}$ with respect to $\mathrm{A}$ in the $5 \mathrm{GHz}$ images between the epochs 1997.6 and 2009.4 and we obtained the results which are graphically demonstrated in Fig. 5 along with the corresponding uncertainties. The separation between $\mathrm{A}$ and $\mathrm{D}$ (8.2 mas) remained nearly the same in both epochs, B moved away from A so that the angular distance between them increased from 4.4 mas to 4.5 mas, whereas $\mathrm{C}$ moved towards $\mathrm{A}$ - the separation between these two decreased from 5.7 mas to 5.5 mas. This combination of displacements cannot be reconciled with the hypothesis that the western part of $0932+075$ is a CSO whose core belongs to the A-complex, because the non-core components of a CSO should either be stationary or, if not, then all of them should move away from the core.

Based on this surprising result, we rejected the hypothesis that the western part of $0932+075$ is a CSO. Consequently, we claim that in the $5 \mathrm{GHz}$ VLBA images (Fig. 1, upper panels) this object only mimics a CSO. 
A. Marecki and A. Sokołowska: Is radio galaxy $0932+075$ a compact symmetric object?
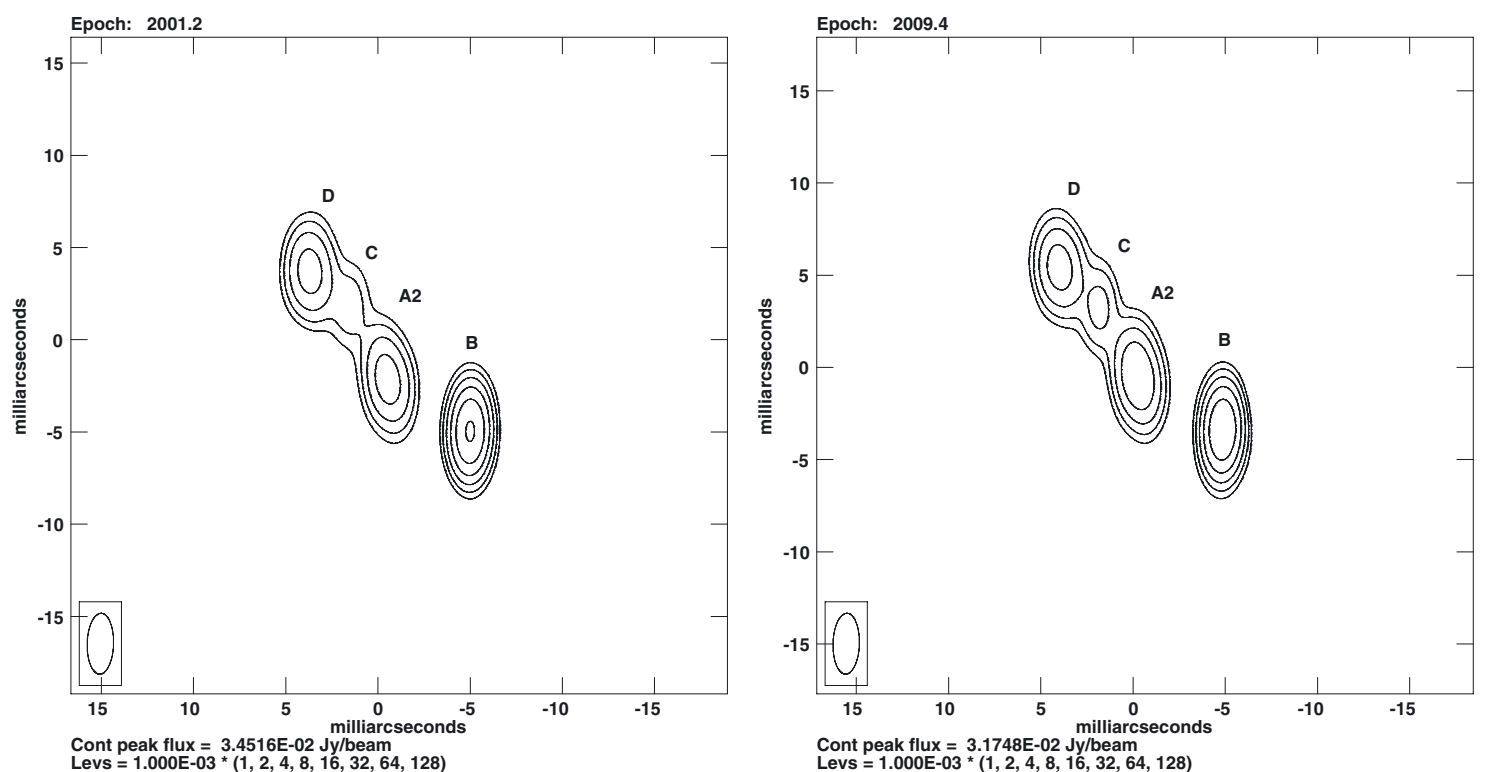

Fig. 6. $15.4 \mathrm{GHz}$ images convolved with the beam of the $5 \mathrm{GHz}$ observation at the epoch 2009.4. Left: the image based on the observation in the epoch 2001.2, right: the image based on the observation in the epoch 2009.4.
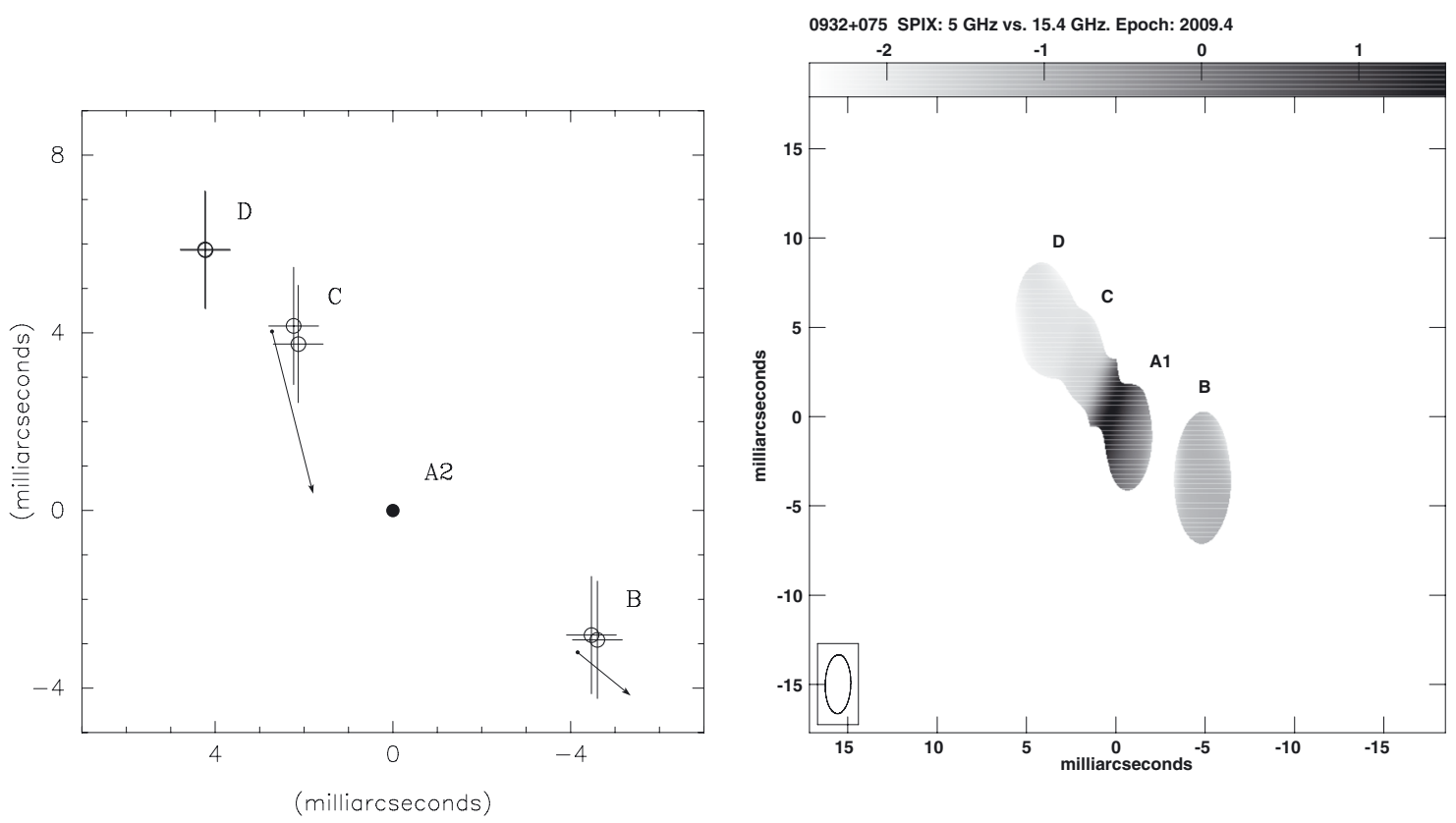

Fig. 7. Left: schematic diagram showing the positions of the four components seen in the $15.4 \mathrm{GHz}$ images convolved with a $5 \mathrm{GHz}$ beam shown in Fig. 6. Open circles represent the positions of the centroids of B, C, and D with respect to A2 in the epochs 2001.2 and 2009.4. Error bars correspond to $20 \%$ of the $5 \mathrm{GHz}$ beam sizes. The arrows indicate the direction of the components' displacements. The length of each arrow is proportional to the magnitude of the respective displacement. Right: $5 \mathrm{GHz}$ vs. $15.4 \mathrm{GHz}$ spectral-index map for epoch 2009.4 prepared from the images shown in Fig. 1 (upper-right panel) and Fig. 6 (right panel).

Given that the result we obtained is non-standard and taking the opportunity that there is another pair of high-resolution observations of $0932+075$, i.e. the $15.4 \mathrm{GHz}$ VLBA observations of epochs 2001.2 and 2009.4 (Fig. 1, lower panels), we attempted a comparison between these two. However, to make the findings from the $15.4 \mathrm{GHz}$ data compatible with the $5 \mathrm{GHz}$ data described above, we convolved both $15.4 \mathrm{GHz}$ images with the beam of one of the $5 \mathrm{GHz}$ images, namely our image of epoch 2009.4. The resulting smeared images are shown in Fig. 6. We fitted Gaussian profiles to the features present in these maps and we measured the changes of the angular distances between component A (we note that it is now co-incident with A2) vs. components B, C, and D to find their displacements. The combined result is shown in Fig. 7 (left panel). The separation between D and A2 remains constant over time which agrees with the $5 \mathrm{GHz}$ measurements. However, quantitatively it differs as it amounts to 7.2 mas, i.e. $\sim 1$ mas less than in the case of the actual $5 \mathrm{GHz}$ maps because A2 in Fig. 7 (left panel) has a different position than the component A in Fig. 5 where it is coincident with A1. For the same reason, the distances A2-B in the set of maps in Fig. 6 are greater by $\sim 1$ mas than those in the true $5 \mathrm{GHz}$ maps and amount to 5.3 mas and 5.4 mas in respective epochs. Furthermore, we have found that the A2-C distance has decreased between epochs 2001.2 and 2009.4 from 4.7 mas 
to 4.3 mas. This decrease confirms our earlier findings of the component $\mathrm{C}$ travelling in the opposite direction to the lobe expansion expected in the case of a CSO. Therefore, we again reached the conclusion that a CSO paradigm is not applicable to the case of $0932+075$. The magnitude of that decrease is twice that measured at $5 \mathrm{GHz}$. This is somewhat uncomfortable, but tolerable given the uncertainties of all the above separations indicated by the error bars in the left panel of Fig. 7: their magnitudes are $20 \%$ of the $5 \mathrm{GHz}$ beam size.

At this stage, we took the opportunity that the $15.4 \mathrm{GHz}$ image of epoch 2009.4 convolved with the $5 \mathrm{GHz}$ beam (Fig. 6, right panel) is ideally suited to be combined with the $5 \mathrm{GHz}$ image of the same epoch (Fig. 1, upper-right panel) to calculate the spectral indices between these two. The resulting spectral-index map is shown in Fig. 7 (right panel). As can be clearly seen there, the area featured by inverted spectrum, i.e. the location of the HFP, is not coincident with the centre of the silhouette of component A1. This is in full agreement with our findings reported in Sect. 2.

Finally, we compared the two original-beam $15.4 \mathrm{GHz}$ images of $0932+075$. Here, the A2-B distance changed from $5.57 \pm 0.60$ mas to $5.68 \pm 0.45$ mas with the uncertainties of $20 \%$ of the $15.4 \mathrm{GHz}$ beam sizes. The difference between these figures remains in agreement with that derived from $5 \mathrm{GHz}$ maps and $15.4 \mathrm{GHz}$ maps convolved with a $5 \mathrm{GHz}$ beam. The A2-C distance has decreased from $4.29 \pm 0.73$ to $4.00 \pm 0.55$. This result is probably not as reliable as it might seem because of the noticeable departure from the Gaussian shape of $\mathrm{C}$ as seen in the epoch 2001.2 map (Fig. 1, lower-left panel) which causes difficulties with fitting a single Gaussian profile. Measuring the angular distance to $\mathrm{D}$ was problematic since it has a double structure at $15.4 \mathrm{GHz}$ (as well as at $22.2 \mathrm{GHz}$ ). We failed to fit a Gaussian model to the component DW in the map from epoch 2001.2. Therefore, we only measured the separation A2-DE that changed from $7.31 \pm 0.71$ mas to $7.37 \pm 0.54$ mas. It follows that, while the $\mathrm{D}$ component as a whole remains stationary with respect to $A$, both at $5 \mathrm{GHz}$ and $15.4 \mathrm{GHz}$, its subcomponents probably move apart. Secondepoch $22.2 \mathrm{GHz}$ observations where DE and DW are only sufficiently well resolved should shed more light on this question.

\section{Conclusions and future work}

Using the observational data presented here, we provided the two main pieces of new evidence characterising 0932+075. First, one of the components of the milliarcsecond-scale structure of this source (A2) has a clearly inverted spectrum for frequencies up to $22 \mathrm{GHz}$. Owing to this property we label it a HFP. Second, it turns out that the western part of $0932+075$ is not a CSO as the $5 \mathrm{GHz}$ VLBA image of epoch 1997.6 (upper-left panel of Fig. 1) might suggest. Instead, we propose the following tentative interpretation of the milliarcsecond-scale morphology of the western part of $0932+075$. What we see in the $15.4 \mathrm{GHz}$ and $22.2 \mathrm{GHz}$ VLBA images is a superposition of two groups of components. The first group consists of A2 and its closest neighbours $\mathrm{A} 1$ and $\mathrm{A} 3$, whereas the second group encompasses all the remaining components. This division was chosen because the second group is nearly co-linear while the A-complex is an outlier. It is not clear what kind of relationship connects the HFP to the other components of $0932+075$ and whether the co-linearity of the latter is significant, but there is a hint that it might be significant given that the directions of the displacements of $\mathrm{B}$ and $\mathrm{C}$ with respect to $\mathrm{D}$ are roughly the same as that of the line connecting these three components (see Fig. 5). It could be thus speculated that the co-linearity is caused simply by the motion of $\mathrm{B}$ and $\mathrm{C}$ both travelling from the origin located at D. However, the weak point of this scenario is that $\mathrm{D}$ plays the role of a secondary core, which is doubtful because of its steep spectrum.

The main drawback of the observational material presented here is that Browne et al. did not use the phase referencing, and neither did we. The reason for this is simple: $0932+075$ itself is an acknowledged phase calibrator used for interferometric observations. Since we have now discovered quite complicated displacements in its milliarcsecond-scale structure, it appears that it would have been very advantageous if the VLBI observations of $0932+075$ of all epochs had been phase referenced to the same phase calibrator so that the absolute positions of the components could have been known. Unfortunately, this is not the case and we had to rely on the measurements of the relative positions as presented in Sect. 3. In addition, the two-point proper motion measurements we have carried out are not very reliable hence the results presented here should be regarded as only preliminary. Consequently, a much more thorough study based on highresolution monitoring of $0932+075$ is necessary to carry out, as accurately as possible, measurements of the proper motions of its milliarcsecond-scale components based on their absolute positions obtained from phase referencing. The new observations should be multi-frequency and quasi-simultaneous with regard to the set of frequencies at a particular epoch to find out what the exact spectral indices of each component are. Full polarisation information would also be helpful since only very few CSO jets have measurable linear polarisation, whereas in blazar jets fractional polarisation tends to increase with distance from the core.

Acknowledgements. The VLBA is operated by the National Radio Astronomy Observatory, a facility of the National Science Foundation operated under cooperative agreement by Associated Universities, Inc. The VLBA observation of $0932+075$ at $15.4 \mathrm{GHz}$ carried out on 22 March 2001 was proposed by the CLASS team, specifically by Neal J. Jackson and Martin Norbury. It was devised to be an ultimate proof that $0932+075$ was not a GL system. However, the image resulting from that observation has never been published. The raw data belongs to the public domain yet we contacted the proposers and we have received written permission to publish the map resulting from that data in the present paper. This research has made use of the NASA/IPAC Extragalactic Database (NED) which is operated by the Jet Propulsion Laboratory, California Institute of Technology, under contract with the National Aeronautics and Space Administration.

\section{References}

An, T., Wu, F., Yang, J., et al. 2012, ApJS, 198, 5

Browne, I. W. A., Wilkinson, P. N., Patnaik, A. R., \& Wrobel, J. M. 1998, MNRAS, 293, 257

Browne, I. W. A., Wilkinson, P. N., Jackson, N. J. F., et al. 2003, MNRAS, 341, 13

Dallacasa, D., Stanghellini, C., Centonza, M., \& Fanti, R. 2000, A\&A, 363, 887 Fanti, C. 2009, Astron. Nachr., 330, 120

Gugliucci, N. E., Taylor, G. B., Peck, A. B., \& Giroletti, M. 2005, ApJ, 622, 136

Marecki, A., Barthel, P. D., Polatidis, A., \& Owsianik, I. 2003, PASA, 20, 16

Myers, S. T., Jackson, N. J., Browne, I. W. A., et al. 2003, MNRAS, 341, 1

Nagai, H., Inoue, M., Asada, K., Kameno, S., \& Doi, A. 2006, ApJ, 648, 148

Owsianik, I., \& Conway, J. E. 1998, A\&A, 337, 69

Owsianik, I., Conway, J. E., \& Polatidis, A. G. 1998, A\&A, 336, L37

Pearson, T. J., \& Readhead, A. C. S. 1988, ApJ, 328, 114

Polatidis, A. G. 2009, Astron. Nachr., 330, 149

Polatidis, A. G., \& Conway, J. E. 2003, PASA, 20, 69

Readhead, A. C. S., Xu, W., Pearson, T. J., Wilkinson, P. N., \& Polatidis, A. G. 1994, NRAO Workshop: Compact Extragalactic Radio Sources, eds. J. A. Zensus, \& K. I. Kellermann, 17

Taylor, G. B., Marr, J. M., Pearson, T. J., \& Readhead, A. C. S. 2000, ApJ, 541, 112

Wilkinson, P. N., Polatidis, A. G., Readhead, A. C. S., Xu, W., \& Pearson, T. J. 1994, ApJ, 432, L87 